\title{
Baden, Feuchtigkeitspflege und Pflege des Windelbereichs
}

\author{
Claudia Dachs, Ulla Busmann, Hans F. Merk
}

\author{
Die Hautpflege bei Neugeborenen und Säuglingen ist ein wichtiges Thema in der Beratung \\ junger Eltern. Angewandte Pflegeroutinen basieren oft auf Traditionen und Erfahrungen. So \\ hat sich eine Vielfalt an Empfehlungen herausgebildet, die Eltern bisweilen überfordert. Auf \\ Basis wissenschaftlicher Untersuchungen von Pflegeroutinen lassen sich Empfehlungen \\ abgeben. Teil 2 der Artikelserie beleuchtet, worauf beim Baden, bei der Feuchtigkeitspflege \\ und bei der Pflege des Windelbereichs zu achten ist.
}

\begin{abstract}
Merke
Alle nachfolgenden Empfehlungen folgen dem Grundsatz: Babyhautpflege sollte die Hautbarriere unterstützen und die Hautreifung nicht stören.
\end{abstract}

\section{Baden von Säuglingen}

Das Säuglingsbad hat positive Effekte. Die Förderung der Eltern-Kind-Bindung ist sicher einer der bedeutsamsten. Es stellen sich jedoch Fragen nach der Auswirkung auf die Haut: Wie oft darf gebadet werden? Ist Baden besser als Waschen? Schaden Badezusätze der Säuglingshaut? Und wie beeinflussen Babypflegeprodukte die Hautschutzbarriere?

In den vergangenen Jahren wurden zahlreiche Studien veröffentlicht, die einigen der häufigsten Fragen der Säuglingshautpflege unter kontrollierten Bedingungen nachgehen. Hervorzuheben sind vor allem drei Untersuchungen aus den Jahren 2009 bis 2011. Zwei von ihnen wurden an der Klinik für Dermatologie, Allergologie und Venerologie der Charité Universitätsmedizin Berlin durchgeführt, die dritte durch das Institut für Hebammenkunde an der britischen Universität Manchester.

\section{Baden oder Waschen?}

Eine Studie der Klinik für Dermatologie, Allergologie und Venerologie der Charité Universitätsmedizin Berlin ging folgender Fragestellung nach: Wie beeinflusst Baden im Unterschied zum Waschen mit einem Waschlappen die natürliche Anpassung und Funktion der Hautschutzbarriere bei gesunden Reifgeborenen in den ersten vier Lebenswochen?

\section{Methodik}

Randomisierung: 57 Neugeborene (Alter $\leq 48$ h) wurden randomisiert-also nach dem Zufallsprinzip-in zwei Gruppen eingeteilt und über die Dauer von 4 Wochen 2mal pro Woche:

- Gruppe A mit klarem Wasser gebadet

- Gruppe B mit klarem Wasser und Waschlappen gewaschen

Kontrollierte Bedingungen: Die optimale Wassertemperatur wurde zwischen 37 und 38 Grad Celsius angesetzt und die Raumtemperatur betrug 22 bis 27 Grad Celsius. Als Badedauer wurden 5 Minuten festgelegt.

Messungen: Die Schlüsselwerte der Funktion der Hautschutzbarriere (TEWL, SCH, pH-Wert) wurden an Stirn, Bauch, Gesäß und Oberschenkel gemessen. Die Messungen wurden an Tag 2, Tag 7 und Tag 28 des Untersuchungszeitraums durchgeführt.

\section{Ergebnisse}

Gebadete zeigten im Vergleich zu gewaschenen Säuglingen

- eine höhere Hautfeuchtigkeit an Stirn und Bauch

- einen signifikant geringeren, transepidermalen Wasserverlust im Windelbereich

\section{Schlussfolgerungen}

2-mal wöchentliches Baden in den ersten Lebenswochen schadet der empfindlichen Haut der Neugeborenen nicht. Es wirkt sich sogar etwas positiver auf die Hautfeuchtigkeit aus als das Waschen mit einem Waschlappen. 
Die Studienergebnisse lassen einen günstigen Langzeiteffekt des Badens auf die Hautschutzbarriere vermuten.

\section{GLOSSAR}

- Stratum corneum: Das Stratum corneum ist die aus Korneozyten bestehende äußerste Schicht der Epidermis (Oberhaut). Das Keratin der Korneozyten sowie die diesen zwischengelagerten Lipide sind essenzielle Bestandteile der Hautschutzbarriere.

- TEWL: Der Transepidermale Wasserverlust (TEWL) bezeichnet den kontinuierlichen Verlust von im Stratum corneum und in der Epidermis gespeicherten Wassers an die Umgebung. Dieser ist auf den Konzentrationsunterschied zwischen Stratum corneum und Umgebungsluft zurückzuführen. Er definiert sich über den Wasserverlust in Gramm pro Quadratmeter Haut pro Stunde. Der Grad des TEWL ist dabei abhängig vom Zustand des Stratum corneums.

- SCH: Die Stratum Corneum Hydration (SCH) bezeichnet den Wasseranteil im Stratum corneum. Eine ausreichende Hydration erschwert das Eindringen von hydrophoben Stoffen in die Haut und ist Voraussetzung für die Aufrechterhaltung der physiologischen Struktur und Barrierefunktion des Stratum Corneums.

- pH-Wert: Der pH-Wert dient als Maß für den sauren oder basischen Charakter einer wässrigen Lösung und definiert die Wasserstoffionen-Konzentration. Ein pH-Wert von 7 ist neutral. Werte darunter gelten als sauer, Werte darüber als basisch. Allgemein besitzt die gesunde Hautoberfläche einen leicht sauren $\mathrm{pH}$-Wert, der die Haut vor Krankheitserregern schützt. Als physiologischen pH-Wert bezeichnet man den Wert, der an der Oberfläche des Unterarms eines gesunden, erwachsenen Mannes gemessen wird. Dieser liegt ca. bei 5,4 bis 5,9. Bei Neugeborenen liegt der pH-Wert zunächst mit circa 7,0 im neutralen Bereich. Er sinkt während der ersten Lebenswoche zunächst schneller, in den folgenden Wochen langsamer ab, um sich letztlich innerhalb des ersten Lebensmonats bei Werten zwischen 5,2 und 5,5 zu stabilisieren.

\section{Babybadezusätze oder klares Wasser?}

Eine Studie der School of Nursing, Midwifery and Social Work an der Universität von Manchester verfolgte die Fragestellung: Hat die Verwendung eines reinigenden, milden Babybadezusatzes beeinträchtigende Wirkungen auf die Haut von Neugeborenen im Vergleich zur Verwendung von klarem Wasser?

\section{Methodik}

Randomisierung: 307 Neugeborene (Alter $\leq 48$ h) wurden randomisiert in zwei Gruppen eingeteilt und 4 Wochen lang je 3-mal pro Woche gebadet, und zwar:

- Gruppe A mit klarem Wasser

- Gruppe B mit einem Babybadezusatz

Messungen: Die Schlüsselwerte der Funktion der Hautschutzbarriere (TEWL, SCH, pH-Wert) wurden an Oberarm, Oberschenkel und Bauch gemessen. Messungen wurden an Tag 2, 14 und 28 des Untersuchungszeitraums durchgeführt.

\section{Ergebnisse}

Es zeigte sich, dass die Zugabe eines sanften Babybadezusatzes gegenüber klarem Wasser

- nicht unterlegen war,

- den transepidermalen Wasserverlust nicht verstärkt und

- keine negativen Effekte auf die empfindliche Säuglingshaut hatte.

\section{Schlussfolgerung}

Speziell für die Reinigung der Säuglingshaut entwickelte Babybadezusätze schaden dieser in keiner Weise.

\section{Die Wirkung von Babypflegeprodukten auf die Hautbarriere}

Eine Studie der Klinik für Dermatologie, Allergologie und Venerologie der Charité Universitätsmedizin Berlin ging folgender Fragestellung nach: Welchen Einfluss haben verschiedene Reinigungs und Pflegeroutinen auf die Hautschutzbarriere von gesunden Reifgeborenen?

\section{Methodik}

Randomisierung: 64 Neugeborene (Alter<48 h) wurden randomisiert in 4 Gruppen eingeteilt und acht Wochen lang je zweimal pro Woche gebadet. Folgende Pflegeroutinen wurden angewandt:

- Gruppe A klares Wasser (= Kontrollgruppe)

- Gruppe B klares Wasser und anschließende Verwendung von Babypflegecreme

- Gruppe C Babywaschgel

- Gruppe D Babywaschgel und anschließende Verwendung von Babypflegecreme

Kontrollierte Bedingungen: Pflegeroutinen und Messungen wurden unter häuslichen Bedingungen durchgeführt. Die Wassertemperatur wurde zwischen 37 und 38 Grad Celsius angesetzt und die Raumtemperatur betrug 22 bis $27 \mathrm{Grad}$ Celsius. Als Badedauer wurden 5 Minuten festgelegt.

Messungen: Die Schlüsselwerte der Funktion der Hautschutzbarriere (TEWL, SCH, pH-Wert) wurden an Stirn, Bauch, Gesäß und Oberschenkel gemessen. Messungen wurden an den Tagen 2, 14, 28 und 56 des Untersuchungszeitraums durchgeführt 


\section{Ergebnisse}

Im Vergleich zur Kontrollgruppe zeigten die Gruppen B und D (Verwendung von Babypflegecreme nach dem Baden)

- einen signifikant geringeren transepidermalen Wasserverlust an Stirn, Bauch und Oberschenkel

- einen höheren Feuchtigkeitsgehalt des Stratum corneum an Stirn und Bauch

\section{Gruppe C zeigte}

- einen leicht erhöhten transepidermalen Wasserverlust, der allerdings nicht signifikant war

- eine schnellere Abnahme des pH-Wertes allein durch die Verwendung des Badezusatzes - dies deutet möglicherweise auf eine schnellere Entwicklung des physiologischen Säureschutzmantels hin

\section{Schlussfolgerungen}

Die 2-mal wöchentliche Verwendung von Babywaschgel und/oder Babypflegecreme verbessert die physiologische Funktion der Hautschutzbarriere im Vergleich zur ausschließlichen Verwendung von klarem Wasser.

\section{FAZIT}

Evidenzbasierte Pflegeempfehlungen für das Baden von Säuglingen

Baden sollte dem Waschen mit einem Waschlappen vorgezogen werden. Wissenschaftlicher Hintergrund: 2-mal wöchentliches Baden in den ersten Lebenswochen schadet der empfindlichen Haut der Neugeborenen nicht. Es wirkt sich sogar etwas positiver auf die Hautfeuchtigkeit aus als das Waschen des Körpers mit einem Waschlappen.

Regelmäßiges Baden von Neugeborenen und Säuglingen: Es wird empfohlen, gesunde Neugeborene und Säuglinge, nach Vorreinigung des Windelbereichs, mindestens 2- bis 3-mal pro Woche zu baden. Wissenschaftlicher Hintergrund: Europäische Pädiater und Dermatologen empfehlen in einem Konsensus-Papier 2- bis 3-mal wöchentliches Baden statt Waschen mit einem Waschlappen.

Beginn der Baderoutine: Mit dem regelmäßigen Baden kann schon begonnen werden, sobald der Nabelschnurrest abgefallen ist. Wissenschaftlicher Hintergrund: Mit dem regelmäßigen Baden sollte nach Abfall des Nabelschnurrestes und Abheilen des Nabelgrundes begonnen werden, insbesondere um Entzündungen zu vermeiden. Der Abfall des Nabelschnurrestes war der Startpunkt für den Beginn des Routinebadens in zwei klinischen Studien mit Neugeborenen an der Klinik für Dermatologie der Charité Universitätsmedizin Berlin.
Wasser- und Raumtemperatur, Badedauer: Der Raum sollte 21 bis 24 Grad warm sein und das Wasser eine Temperatur zwischen 37 und maximal 37,5 Grad Celsius haben. Die Badedauer sollte sich auf 5 bis 10 Minuten beschränken. Das Baby sollte schnell, aber sanft abgetrocknet werden (abtupfen, nicht reiben), damit es nach dem Baden nicht auskühlt. Wissenschaftlicher Hintergrund: Für ihre Studie über das Baden von Neugeborenen und Säuglingen arbeiteten Forscher der Klinik für Dermatologie, Allergologie und Venerologie der Charité Universitätsmedizin Berlin mit folgenden Richtwerten: Die Wassertemperatur wurde zwischen 37 und 38 Grad Celsius angesetzt und die Raumtemperatur betrug 22 bis 27 Grad Celsius. Als Badedauer wurden 5 Minuten festgelegt. Ein europäisches KonsensusPapier empfiehlt eine Badedauer von 5 bis 10 Minuten. Unmittelbar nach dem Bad sollte das Kind mit einem Handtuch bedeckt und trocken getupft, auf keinen Fall aber trocken gerieben werden.

Einsatz von Badezusätzen. Der Einsatz eines milden Babybadezusatzes beeinträchtigt die Entwicklung der Säuglingshaut nicht und ist für das Baden gesunder Säuglinge ebenso empfehlenswert wie klares Wasser. Wissenschaftlicher Hintergrund: Milde Badezusätze für die Säuglingspflege reinigen die Haut auch von fettlöslichen Substanzen. Dies gelingt nicht mit Wasser allein.

Richtige Wahl des Badezusatzes. Ein milder flüssiger Badezusatz, der speziell für die Bedürfnisse von Säuglingshaut entwickelt worden ist, kann für die schonende Reinigung beim Baden eingesetzt werden. Wissenschaftlicher Hintergrund: Die Hautschutzbarriere von Säuglingen unterscheidet sich im ersten Lebensjahr und auch darüber hinaus von jener der Erwachsenen. Sie ist zum Beispiel etwa 30 Prozent dünner und wesentlich durchlässiger, sodass sie schneller austrocknet. Diesem Umstand sollte mit speziell für die Bedürfnisse von Säuglingshaut entwickelten Produkten Rechnung getragen werden. Ein Einsatz von flüssigen Badezusätzen wird gegenüber Seifenstücken empfohlen. Letztere sind üblicherweise alkalisch und können dadurch, vor allem in Verbindung mit hartem Wasser, die Säuglingshaut reizen und austrocknen. Feuchtigkeitsspendende Inhaltsstoffe können zusätzlichen Schutz für die Säuglingshaut bieten.

Literatur

[1] Anderson GC, Lane AE, Chang HP. Axillary temperature in transitional newborn infants before and after tub bath. Appl Nurs Res 1995; 8: 123-128 
[2] AWHONN. Neonatal Skin Care Evidence-Based Clinical Practice Guideline. 3 rd ed. Washington DC, USA; 2013. ISBN 9781-938299-03-2

[3] Blume-Peytavi U, Cork MJ, Faergemann J et al. Bathing and cleansing in newborns from day 1 to first year of life: recommendations from a European round table meeting. J Eur Acad Der-mata! Venereol 2009; 23: 751-759

[4] Blume-Peytavi U, Hauser M, Stamatas GN et al. Skin care practices for newborns and infants: review of the clinical evidence for best practices. Pediatr Dermatol 2012; 29: 1-14

[5] Bryantonj, Walsh D, Barrett $M$ et al. Tub bathing versus traditional sponge bathing for the newborn. J Obstet Gynecol Neonatal Nurs 2004; 33: 704-712

[6] Darmstadt GL, Dinulos JG. Neonatal skincare. Pediatr Clin North Am 2000; 47: 757-782

[7] Dizon MV, Galzote C, Estanislao R et al. Tolerance of baby cleansers in infants: a randomized controlled trial. Indian Pediatr 2010; 47: 959-963

[8] Garcia Bartels N, Mleczko A, Schink T et al. Influence of bathing or washing on skin barrier function in newborns during the first four weeks of life. Skin Pharmacol Physiol 2009; 22: 248-257

[9] Garcia Bartels N, Scheufele R, Prosch F et al. Effect of standardized skin care regimens on neonatal skin barrier function in different body areas. Pediatr Dermatol 2010; 27: 1-8

[10] Gfatter R, Hackl P, Braun F. Effects of soap and detergents on skin surface $\mathrm{pH}$, stratum corneum hydration and fat content in infants. Dermatology 1997; 195: 258-262

[11] Ghadially R, Halkier-Sorensen L, Elias PM. Effects of petrolatum an stratum corneum structure and function. J Am Acad Dermatol 1992; 26: 387-396

[12] Henningsson A, Nystrom B, Tunnell R. Bathing or washing babies after birth? Lancet 1981; 2: 1401-1403

[13] Lavender T, Bedwell C, O’Brien E et al. Infant skin-cleansing product versus water: a pilot randomized, assessor-blinded controlled trial. BMC Pediatr 2011; 11: 35

[14] Lavender T, Bedwell C, Roberts SA et al. Randomized controlled trial evaluating a baby wash product on skin barrier function in healthy, term neonates. J Obstet Gynecol Neonatal Nurs 2013; 42: 203-214

[15] Medves JM, O'Brien B. The effect of bather and location of first bath on maintaining thermal stability in newborns. J Obstet Gynecol Neonatal Nurs 2004; 33: 175-182

[16] Nako Y, Harigaya A, Tomomasa T et al. Effects of bathing immediately after birth on early neonatal adaptation and morbidity: a prospective randomized comparative study. Pediatr Int 2000; 42:517-522

[17] Penny-MacGillivray T. A newborn's first bath: when? J Obstet Gynecol Neonatal Nurs 1996; 25: 481-487

\section{Feuchtigkeitspflege für Säuglinge}

Der intensive Wasserkontakt beim Baden ist für die Hautbarriere eine besondere Herausforderung. Für Säuglinge gilt dies umso mehr. Daher haben verschiedene Studien diese Belastungssituation in den Mittelpunkt gerückt.

\section{Feuchtigkeitspflege nach dem Bad}

Eine Studie der Klinik für Dermatologie, Allergologie und Venerologie der Charité Universitätsmedizin Berlin zum Einfluss verschiedener Reinigungs- und
Pflegeroutinen auf die Hautschutzbarriere ging u.a. der Fragestellung nach: Wie wirken Säuglingspflegeprodukte auf die physiologische Barrierefunktion der Haut nach dem Baden?

\section{Methodik}

64 Neugeborene (Alter $<48$ h) wurden randomisiert in 4 Gruppen eingeteilt und 8 Wochen lang je 2-mal pro Woche gebadet:

- Gruppe A klares Wasser (= Kontrollgruppe)

- Gruppe B klares Wasser und anschließende Verwendung von Babypflegecreme

- Gruppe C Babywaschgel

- Gruppe D Babywaschgel und anschließende Verwendung von Babypflegecreme

Kontrollierte Bedingungen: Pflegeroutinen und Messungen wurden unter häuslichen Bedingungen durchgeführt. Die Wassertemperatur wurde zwischen 37 und 38 Grad Celsius angesetzt und die Raumtemperatur betrug 22 bis 27 Grad Celsius. Als Badedauer wurden 5 Minuten festgelegt.

Messungen: Die Schlüsselwerte der Funktion der Hautschutzbarriere (TEWL, SCH, pH-Wert) wurden an Stirn, Bauch, Gesäß und Oberschenkel gemessen. Messungen wurden an Tag 2, Tag 14, Tag 28 und Tag 56 des Untersuchungszeitraums durchgeführt.

\section{Ergebnisse}

Im Vergleich zur Kontrollgruppe zeigten die Gruppen $B$ und D (nach anschließender Verwendung einer Babypflegecreme)

- einen signifikant geringeren transepidermalen Wasserverlust an Stirn, Bauch und Oberschenkel

- einen höheren Feuchtigkeitsgehalt des Stratum corneum an Stirn und Bauch

\section{Schlussfolgerungen}

Der Einsatz einer Babypflegecreme nach dem Bad erhöht den Feuchtigkeitsgehalt und verringert den Wasserverlust der Säuglingshaut. Die Effektivität der physiologischen Barrierefunktion wird gesteigert.

\section{Feuchtigkeitspflege nach dem Babyschwimmen}

Eine Studie der Klinik für Dermatologie, Allergologie und Venerologie der Charité Universitätsmedizin Berlin verfolgte die Fragestellung: Wie wirkt sich Babyschwimmen auf die Barrierefunktion der Säuglingshaut aus? Können Pflegeprodukte hier stabilisierend wirken?

\section{Methodik}

Randomisierung: 44 Säuglinge (Alter zwischen 3 und 6 Monaten), die in vier aufeinanderfolgenden Wochen je 1-mal pro Woche an einem Babyschwimmkurs teilnahmen, wurden randomisiert in 2 Gruppen aufgeteilt: 
- Gruppe A abtrocknen nach dem Schwimmen

- Gruppe B abtrocknen nach dem Schwimmen, anschließendes Eincremen mit einer Babypflegelotion

Kontrollierte Bedingungen: Die Kinder waren jeweils 25 bis 40 Minuten im Wasser.

Messungen: TEWL, SCH, pH-Wert und Hautoberflächenlipide wurden an Stirn, Bauch, Po und Oberschenkel gemessen. Messungen wurden vor Beginn der Studie, über die Dauer der Studie jeweils vor dem Schwimmkurs sowie eine Woche nach dem letzten Kurstermin durchgeführt.

\section{Ergebnisse}

Unter Verwendung einer Feuchtigkeitspflege nach dem Babyschwimmen blieben die relevanten Hautparameterinsbesondere der $\mathrm{pH}$-Wert-weitgehend stabil.

In einem Nebeneffekt zeigte sich, dass die eingecremten Säuglinge auch weniger an Infekten litten. Zur Überprüfung eines möglichen Zusammenhangs dieser beiden Effekte bedarf es jedoch weiterer Untersuchungen.

\section{Schlussfolgerungen}

Babyschwimmen schadet der Säuglingshaut nicht. Das anschließende Eincremen kann die relevanten Hautparameter stabil halten - und unter Umständen sogar verbessern. Die Studie hat die positiven Effekte des Eincremens auf die Hautbarriere von Säuglingen nachgewiesen.

\section{FAZIT}

\section{Evidenzbasierte Pflegeempfehlungen für die} Feuchtigkeitspflege

Nutzen von Feuchtigkeitspflege: Speziell für die Pflege der Säuglingshaut entwickelte Feuchtigkeitscremes oder -lotionen können helfen, die Funktion der Hautschutzbarriere zu erhalten oder sogar zu verbessern. Wissenschaftlicher Hintergrund: Im Rahmen einer Studie zur Auswirkung unterschiedlicher Pflegeregimes auf die Funktion der Hautbarriere zeigte die Haut von Neugeborenen und Säuglingen nach dem Eincremen einen höheren Feuchtigkeitsgehalt $(\mathrm{SCH})$ und einen geringeren transepidermalen Wasserverlust (TEWL) als bei Kindern, die nicht eingecremt wurden. Die Babypflegecreme spendete der Haut somit Feuchtigkeit und schützte die natürliche Hautschutzbarriere.

Regelmäßige Feuchtigkeitspflege: Es wird empfohlen, Säuglinge mindestens 2-mal wöchentlich, idealerweise nach dem Baden, einzucremen. Wissenschaftlicher Hintergrund: Die Verwendung einer speziellen Babypflegecreme 2-mal pro Woche verbesserte in einer klinischen Studie an der Hautklinik der Charité Universitätsmedizin Berlin die physiologische Funktion der
Hautschutzbarriere bei Neugeborenen und Säuglingen.

Anwendung der Feuchtigkeitspflege: Pflegeprodukte sollten dünn aufgetragen werden. Wissenschaftlicher Hintergrund: Das Ansammeln zu reichhaltig aufgetragener Pflegeprodukte in Hautfalten sollte vermieden werden. Dies könnte zu einem okklusiven Effekt mit möglichem Einfluss auf die Funktion der Hautbarriere führen.

\section{Literatur}

[18] Blume-Peytavi U, Cork M], Faergemann J et al. Bathing and cleansing in newborns from day 1 to first year of life: recommendations from a European round table meeting. J Eur Acad Der-matol Venereol 2009; 23: 751-759

[19] Danby SG, Bedwell C, Cork M. Neonatal Skincare and Toxicology, Ch. 5. In: Eichenfield LF, Frieden IJ, Mathes EF, Zaenglein AL, eds. Neonatal and Infant Dermatology, 3rd ed. Philadelphia: Saunders; 2015: 46-56. ISBN 978-1-4557-2638-7

[20] Darmstadt CL, Badrawi N, Law PA et al. Topically applied sunflower seed oil prevents invasive bacterial infections in preterm infants in Egypt: a randomized, controlled clinical trial. Pediatr Infect Dis J 2004; 23: 719-725

[21] Darmstadt CL, Saha SK, Ahmed AS et al. Effect of skin barrier therapy on neonatal mortality rates in preterm infants in Bangladesh: a randomized, controlled, clinical trial. Pediatrics 2008; 121: 522-529

[22] Darmstadt CL, Saha SK, Ahmed AS et al. Effect of topical treatment with skin barrier-enhancing emollients on nosocomial infections in preterm infants in Bangladesh: a randomised controlled trial. Lancet 2005; 365: 1039-1045

[23] Denda M. Epidermal proliferative response induced by sodium dodecyl sulphate varies with environmental humidity. $\mathrm{Br}$ J Dermatol 2001; 145: 252-257

[24] Garcia Bartels N, Rosler S, Martus P et al. Effect of baby swimming and baby lotion on the skin barrier of infants aged 3-6 months. J Dtsch Dermatol Ges 2011; 9: 1018-1025

[25] Garcia Bartels N, Scheufele R, Prosch F et al. Effect of standardized skin care regimens on neonatal skin barrier function in different body areas. Pediatr Dermatol 2010; 27: 1-8

[26] larkowski LE, Tierney NK, Horowitz P. Tolerance of skin care regimen in healthy, full-term neonates. Clin Cosmet Investig Dermatol 2013; 6: 137-144

[27] Schurer N, Schliep V, Williams ML. Differential utilization of linoleic and arachidonic acid by cultured human keratinocytes. Skin Pharmacol 1995; 8: 30-40

[28] Telofski LS, Morello AP 3rd, Mack Correa MC et al. The infant skin barrier: can we preserve, protect, and enhance the barrier? Dermatol Res Pract 2012; 2012: 198789

[29] Tsai TF, Maibach HI. How irritant is water? An overview. Contact Dermatitis 1999; 41: 311-314

[30] Uter W, Gefeller 0, Schwanitz H]. An epidemiological study of the influence of season (cold and dry air) on the occurrence of irritant skin changes of the hands. $\mathrm{Br} J$ Dermatol 1998; 138: $266-272$

[31] Willis I.The effects of prolonged water exposure on human skin. J Invest Dermatol 1973; 60: 166-171 


\section{Pflege des Windelbereichs}

Auch wenn, statistisch gesehen, nahezu jeder Säugling in seinem 1. Lebensjahr mindestens $1 \mathrm{mal}$ unter einer Windeldermatitis leidet, ist diese dennoch keineswegs harmlos. Sie verursacht Schmerzen und kann eine Reihe von Komplikationen nach sich ziehen. Bei der Pflege des Windelbereichs ist daher große Sorgfalt angezeigt.

\section{Entstehung der Windeldermatitis}

Zu den physiologischen Besonderheiten der Säuglingshaut gehört, neben der deutlich geringeren Dicke und einer lockereren Struktur als bei Erwachsenenhaut, der deutlich schnellere Wasseraustausch. So kann die Haut empfindlicher gegenüber Keimen und Reizstoffen sein, da der Säureschutzmantel noch nicht so ausgeprägt ist wie bei der Haut eines Erwachsenen.

Untersuchungen zu Struktur und Zusammensetzung der Hautschutzbarriere in verschiedenen Körperzonen haben eine weitere Differenzierung dieser Unterschiede besonders im Windelbereich gezeigt: Der Feuchtigkeitsgehalt des Stratum corneum $(\mathrm{SCH})$ und der pH-Wert gesunder Säuglingshaut sind hier höher. Dies kann in einem Bereich, der besonderen physikalischen und chemischen Belastungen ausgesetzt ist, zu einer Beeinträchtigung der Barrierefunktion führen. Eine Windeldermatitis ist häufig die Folge.

Ratschläge und Vorgehensweisen zur Vorbeugung beziehungsweise Therapie von Windeldermatitiden sind zahlreich. Wissenschaftliche Untersuchungen dazu gibt es hingegen wenige.

\section{Hautpflege im Windelbereich bei Säuglingen zwischen 7 und 11 Monaten}

Eine Studie der Klinik für Dermatologie, Allergologie und Venerologie der Charité Universitätsmedizin Berlin verfolgte diese Fragestellung: Wie wirken unterschiedliche Pflegeregimes auf die physiologische Barrierefunktion der Haut im Windelbereich?

\section{Methodik}

Randomisierung: 83 Säuglinge (Alter 7 bis 11 Monate) wurden randomisiert in 3 Gruppen aufgeteilt. In jedem der 3 Studienarme wurde über die Dauer von 8 Wochen ein anderes Pflegeregime auf den Windelbereich angewendet:

- Gruppe A Reinigung mit Waschlappen und Wasser (= Kontrollgruppe)

- Gruppe B Reinigung mit Waschlappen und Wasser, dann Auftragen einer Wundschutzcreme

- Gruppe C Reinigung mit Feuchttüchern, dann Auftragen einer Wundschutzcreme 
Der Windelwechsel wurde in allen Gruppen mindestens 4-mal innerhalb von 24 Stunden durchgeführt.

Kontrollierte Bedingungen: Alle Gruppen wurden gemäß den evidenzbasierten Pflegeempfehlungen für Neugeborene 2-mal pro Woche mit einem milden Babybadezusatz gebadet und anschließend eingecremt. Der Windelbereich wurde dabei ausgespart.

Messungen: Die Schlüsselwerte der Funktion der Hautschutzbarriere (TEWL, SCH, pH-Wert) wurden an Bauch, Oberschenkel und Gesäß gemessen. Messungen wurden an Tag 2, 14 und 28 des Untersuchungszeitraums durchgeführt.

\section{Ergebnisse}

- Keines der untersuchten Pflegeregimes für die Haut und den Windelbereich hat die Hautschutzbarriere negativ beeinflusst.

- In keiner der 3 Gruppen trat vermehrt Windeldermatitis auf. Häufigkeit und Verlauf waren vergleichbar und altersentsprechend.

- In den Gruppen B und C-hier kam Wundschutzcreme zum Einsatz-lagen die reduzierten SCHWerte signifikant über denen außerhalb der Windelregion. Das bedeutet: Der Feuchtigkeitsgehalt der gesunden Haut im Windelbereich konnte gesenkt werden.

\section{Schlussfolgerungen}

Die Charité-Studie hat die unterstützende Wirkung der Hautpflegeregimes für Neugeborene erstmals auch für Säuglinge von 7 bis 11 Monaten nachgewiesen. Darüber hinaus ist die Haut im Windelbereich besonderen Belastungen ausgesetzt. Sie sollte sorgfältig gepflegt werden, um einer Windeldermatitis bestmöglich vorbeugen zu können.

\section{Hautpflege bei Windeldermatitis mit Muttermilch oder Wundschutzcreme}

Eine Studie der Department of Pediatric Nursing, Faculty of Nursing der Universität Istanbul verglich die Behandlung von Windeldermatitis bei Neugeborenen hinsichtlich der Fragestellung: Muttermilch oder Wundschutzcreme?

\section{Methodik}

Randomisierung: 63 Neugeborene, die während des Aufenthalts in der Klinik eine Windeldermatitis entwickelten, wurden in 2 Gruppen eingeteilt. In beiden Studienarmen wurde über die Dauer von 5 Tagen eine andere Behandlung des Windelbereichs durchgeführt:

- Gruppe A Auftragen von Muttermilch auf den betroffenen Bereich

- Gruppe B Auftragen von Wundschutzcreme (Inhaltsstoffe unter anderem: 40 Prozent Zinkoxid und Lebertran) auf den betroffenen Bereich
Der Windelwechsel wurde in beiden Gruppen alle 3 Stunden durchgeführt. Die Substanzen wurden jeweils während des Windelwechsels aufgetragen.

Die Gruppen zeigten keine statistisch relevanten Unterschiede, weder im Hinblick auf Geschlecht, Gestationsalter, Ernährung oder Reinigungsmethode noch auf andere Faktoren.

Messung: Vor der Behandlung wurden Rötung beziehungsweise Schädigung der Haut anhand einer Skala von 0 bis 3 beurteilt:

- $0=$ nicht betroffen

- 1 = leichtes Erythem

- 2 = großflächiges Erythem

- 3 = großfächiges und tiefer reichendes Erythem

Jeweils 5 Tage lang notierten die Untersucher täglich die Veränderungen des Hautzustands. Die abschließende Beurteilung folgte der zu Beginn verwendeten 4-stufigen Skala. Die Stufe 0 bezeichnete eine „vollständige Heilung“.

\section{Ergebnisse}

- Zwischen den Probanden aus beiden Gruppen, deren Hautzustand zu Studienbeginn mit der Stufe 1 bewertet wurde, zeigten sich keine statistisch signifikanten Unterschiede in der Verbesserung des Hautzustands.

- Bei jenen Kindern, deren Grad der Rötung zu Beginn auf 2 oder 3 gesetzt war, führte Wundschutzcreme signifikant zu besseren Fortschritten als Muttermilch.

- Bei reifgeborenen Kindern unter Antibiotikabehandlung sowie bei ausschließlich gestillten Neugeborenen führte die Verwendung von Wundschutzcreme ebenfalls zu signifikant besseren Ergebnissen.

\section{Schlussfolgerungen}

Die verwendete Wundschutzcreme mit Zinkoxid und Lebertran wirkte effektiver gegen Windeldermatitis als Muttermilch. Dies gilt besonders für Neugeborene, deren Windelbereich mittelstark bis stark betroffen ist. Bei nur leicht geröteter Haut sind beide Behandlungsmethoden ähnlich erfolgreich.

FAZIT

Evidenzbasierte Empfehlungen für die Pflege des Windelbereichs

Häufiger Windelwechsel: Windeln sollten so oft wie nötig gewechselt werden, um sicherzustellen, dass die Haut sauber und trocken gehalten wird. Wissenschaftlicher Hintergrund: Das Tragen feuchter Materialien (Stoff oder Einwegwindeln) auf der Haut 
führt zu erhöhter Hautfeuchtigkeit. Diese hat ihrerseits negativen Einfluss auf die Empfindlichkeit der Haut gegenüber Reibung und entsprechender Erosion, ihre Durchlässigkeit und ihre bakterielle Besiedelung. Dies konnte in einem Modellversuch an der Unterarmhaut von Erwachsenen gezeigt werden.

Sanfte Reinigung: Der Windelbereich sollte sanft gereinigt werden - entweder mit einem Waschlappen beziehungsweise Baumwollwatte und Wasser alleine oder mit einem feuchten Babypflegetuch. Wissenschaftlicher Hintergrund: In einer Studie der School of Nursing, Midwifery and Social Work an der Universität von Manchester wurde die Reinigung des Windelbereichs mit parfümfreien Babypflegetüchern mit der Reinigung mit Wasser und Baumwollwatte verglichen. Beide Methoden zeigten keine Unterschiede im Einfluss auf die Schlüsselwerte der Funktion der Hautschutzbarriere.

Gründlich und vorsichtig trocken tupfen: Der Windelbereich sollte gründlich, aber vorsichtig trocken getupft werden. Anschließend sollte das Kind bei Raumtemperatur an der Luft strampeln dürfen. Wissenschaftlicher Hintergrund: Da Reibung zu Irritationen beziehungsweise Schädigung der

Hautschutzbarriere führen kann, sollte die Haut entweder durch sanftes Tupfen mit einem weichen Tuch beziehungsweise Watte oder durch Lufteinwirkung getrocknet werden (kein Föhnen!).

\section{Literatur}

[32] Adam R, Sehnetz B, Mathey P et al. Clinical demonstration of skin mildness and suitability for sensitive infant skin of a new baby wipe. Pediatr Dermatol 2009; 26: 506-513

[33] Andersen PH, Bucher AP, Saeed I et al. Faecal enzymes: in vivo human skin irritation. Contact Dermatitis 1994; 30: 152-158

[34] Atherton D, Mills K. What can be done to keep babies' skin healthy? RCM Midwives 2004; 7: 288-290

[35] AWHONN. Neonatal Skin Care Evidence-Based Clinical Practice Guideline. 3 rd ed. Washington DC, USA. 2013. Washington DC, USA, ISBN 978-1-938299-03-2

[36] Blume-Peytavi U, Hauser M, Lünnemann L et al. Prevention of Diaper Dermatitis in Infants - a Literature Review. Pediatr Dermatol 2014; 31: 413-429

[37] Danby SG, Bedwell C, Cork M. Neonatal Skin Care and Toxicology. In: Eichenfield LF, Frieden IJ, Mathes EF, Zaenglein AL, eds. Neonatal and Infant Dermatology, 3rd ect. Philadelphia: Saunders; 2015: 46-56. ISBN 978-1-4557-2638-7

[38] Ehretsmann C, Schaefer P, Adam R. Cutaneous tolerance of baby wipes by infants with atopic dermatitis, and comparison of the mildness of baby wipe and water in infant skin. J Eur Acad Dermatol Venereol 2001; 15 (Suppl 1): 16-21

[39] Garcia Bartels N, Massoudy L, Scheufele R et al. Standardized diaper care regimen: a prospective, randomized pilot study on skin barrier function and epidermal IL-l $\alpha$ in newboms. Pediatr Dermatol 2012; 29: 270-276
[40] Jackson PD. Diaper dermatitis. Protecting the bottom line. Adv Nurse Pract 2010; 18: 35-36, 38-41

[41] Lavender T, Furber C, Campbell $\mathrm{M}$ et al. Effect on skin hydration of using baby wipes to clean the napkin area of newborn babies: assessor-blinded randomised controlled equivalence trial. BMC Pediatr 2012; 12: 59

[42] Priestley GC, McVittie E, Aldridge RD. Changes in skin pH after the use of baby wipes. PediatrDermatol 1996; 13: 14-17

[43] Senses DA, Ozturk CE, Yar NE et al. Do baby wet wipes change periurethral aerobic flora? Jpn J Infect Dis 2007; 60: 225-226

[44] Shin HT. Diaper dermatitis that does not quit. Dermatol Ther 2005; 18: 124-135

[45] Visscher M, Odio M, Taylor T et al. Skin care in the NICU patient: effects of wipes versus cloth and water on stratum corneum integrity. Neonatology 2009; 96: 226-234

[46] Zimmerer RE, Lawson KD, Calvert C].The effects of wearing diapers on skin. Pediatr Dermatol1986; 3: 95-101

\section{VORSCHAU}

Teil 3 der Artikelserie in Ausgabe 5/ 2018 nimmt Produkte für die Pflege der Säuglingshaut unter die Lupe, verrät, wie Öle in der Säuglingspflege sich auf die Hautschutzbarriere auswirken, erklärt, welche Inhaltsstoffe in Pflegeprodukten enthalten sind, und kommt so zu Empfehlungen zur Beschaffenheit von Reinigungs- und Pflegeprodukten für die Säuglingshaut.

\section{Autorinnen / Autoren}

Ulla Busmann ist Dipl.-Pflegepädagogin und stellvertretende Schulleiterin an der Kath. Schule für Pflegeberufe Essen $\mathrm{gGmbH}$ in Essen.

Claudia Dachs ist Dipl. Pflegepädagogin, Hebamme und Anbieterin von Kursen zur Babypflege in Kleinaitingen.

Prof. Dr. Hans F. Merk ist Dermatologe in Aachen und war mehr als 20 Jahre lang als Direktor der Aachener Hautklinik tätig.

\section{Korrespondenzadresse}

Univ.-Prof. Dr. med. Hans F. Merk

Direktor (em.) Klinik für Dermatologie \& Allergologie RWTH Aachen University

E-Mail: hans.merk@post.rwth-aachen.de

\section{Über diesen Artikel}

Die dreiteilige Artikel-Serie zur Pflege der Haut von Neugeborenen und Säuglingen basiert auf dem Handbuch für die Hebammenpraxis „Evidenzbasierte Pflege der Säuglingshaut“ von Claudia Dachs, Ulla Busmann und Prof. Dr. Hans F. Merk, Stuttgart 2016, Thieme 